\title{
Clinician opioid prescribing practices and patient utilization of prescribed opioids in pediatrics
}

Jeannie S. Huang, MD, MPH; Cynthia L. Kuelbs, MD

\section{ARTICLE INFO}

Keywords:

opioids

children

adolescents

prescriptions

pain

DOI:10.5055/jom.2018.0463

(C) 2018 Journal of Opioid Management, All Rights Reserved.

\begin{abstract}
Objective: Little is known regarding clinician prescribing of opioid medications and of patient use of prescribed opioid medications in pediatrics. The authors sought to learn more about pediatric clinician opioid prescribing practices and patient utilization and disposal of prescribed opioids.

Design: Cross-sectional, observational study.

Setting: Tertiary care pediatric healthcare center.

Participants: Pediatric clinicians who prescribe opioid medications and parents of children prescribed an opioid medication.
\end{abstract}

Main Outcome Measures: Clinicians were surveyed about opioid prescribing practices for acute pain management in children, and parents were asked about utilization and disposal of prescribed opioids.

Results: Most clinician respondents (64 percent) reported prescribing opioid medications to manage acute pain. The typical length of opioid prescriptions was limited to 7 days (93 percent). Parents reported a high prevalence of leftover opioid medications (86 percent). Most (59 percent) did not dispose of the remaining medication.

Conclusions: Targets for intervention to reduce unnecessary opioid exposure in youth are identified.
Recent data highlight morbidity and adverse outcomes from pediatric opioid exposure and the contributory role of clinicians to the development of opioid misuse in adolescence and adulthood. Adolescents may be particularly vulnerable to the addictive properties and adverse outcomes associated with opioids. First, use of other addictive substances (particularly underage alcohol and drug use) is already prevalent among adolescents. ${ }^{1}$ Second, opioid misuse has been linked with dating violence and legal problems in adolescent and young adult users. ${ }^{2}$ Although the current opioid crisis primarily affects adults and older adolescents, increasing evidence suggests that opiates should be used with caution in younger children as well. For example, adverse effects on fetal brain development from gestational prescription opioid exposure ${ }^{3,4}$ have been demonstrated. Additionally, pediatric exposure to prescribed opioid medications has been linked with future adult opioid misuse, ${ }^{5}$ and medical use of prescription opioids commonly precedes nonprescription opioid use. ${ }^{6}$ A recent study demonstrated a small (3-15 percent) but present risk for persistent opioid use in adolescents and young adults following surgery (defined as still using opioids 3-6 mo after surgery). ${ }^{7}$ Further, individuals with an age of onset of opioid use younger than 18 years are at higher odds for having a physical or psychiatric comorbid disorder as compared to persons who began opioid use when 31 years or older. ${ }^{8}$

Despite current scrutiny of clinician prescribing practices as contributory to opioid misuse, little is known about pediatric clinicians' opioid prescribing practices for acute pain management and how these medications are actually used by parents for acute management of their child's pain. Given the aforementioned potential risks of pediatric opioid exposure as well as the risks posed for overdose 
and misuse when prescribed and unused opioids are left in the home, we sought to add to the literature by learning more about pediatric clinician opioid prescribing practices and patient utilization and disposal of prescribed opioids.

\section{METHODS}

As part of an institutional quality improvement project to improve patient safety outcomes related to opioid treatment, surveys (see Tables 1 and 2) were distributed to clinicians practicing at a pediatric tertiary care center of a major metropolitan region serving $>1$ million children (as determined by medical staff membership with an active license to prescribe opioids) and to parents of children at that center who had received an opioid prescription within the past 3.5 months (based on prescription records from November 1, 2016 to February 13, 2017). Invitations to participate in respective surveys were e-mailed to targeted participants with a link to an online survey. Eight hundred eighty-seven physicians and 117 nurse practitioners and physician assistants with licenses to prescribe opioids were sent the clinician survey, and 772 parents were sent the parent survey. Survey responses were not tied to any identifiable personal information. Responses were reported as actual number of responses in a given category, and relative percentages were calculated and reported to the nearest whole number. Descriptive statistics were performed on survey responses using JMP software (v. 12; Cary, NC). Response categories are listed with corresponding percentages in Tables 1 and 2.

\section{RESULTS}

One hundred ninety active clinicians with opioid prescribing capability completed the survey, and 165 parents completed the parent survey. Survey response rates were 19 percent for clinicians and 21 percent for parents. Survey questions and associated responses are reported in Tables 1 and 2 .

\section{Clinician survey}

Clinician survey questions and responses are reported in Table 1 . The majority of clinician respondents (64 percent) reported dosing opioid medications to manage acute pain. Of 92 responding clinicians who provided specialty data, 66 represented medical ( 72 percent) and 26 represented
(28 percent) surgical subspecialties. The typical length of opioid prescriptions varied substantially but generally was limited to within 7 days (93 percent). Among those using a quantified pain scale to assess pain, the pain threshold for opioid medication prescribing varied between 4 and 10 on a scale of 0 to 10. Thirty-one percent of clinicians evaluated pain based on "clinical expertise and impression of patient pain."

Only a minority of clinicians (25 percent) reported providing prescription refills for acute pain. Among clinicians who refilled opioid medication prescriptions, only a minority (19 percent) reassessed patients' pain prior to the prescription refill using a pain rating scale. Instead, the majority (81 percent) refilled prescriptions based only on patients' report of presence of pain.

Most clinicians (64 percent) reported not having a standard protocol for pain management in their practice setting. Naloxone was generally not prescribed ( 75 percent) in the setting of an opioid prescription. Formal education on pain assessment management had been received by a majority of clinicians (61 percent), but a substantial proportion (39 percent) reported inability to recall or never having received any education on pain assessment/ management.

\section{Parent survey}

Parent survey questions and responses are reported in Table 2 . The majority of parent respondents (93 percent) filled opioid prescriptions when prescribed. Among those who did not fill the prescription, the majority reported that the main reason for not filling the prescription was that the medication was not needed. For those who took opioid medications, most children took the medication for 3 days at most, if at all ( 6 percent did not take any), and 86 percent had leftover prescription pain medication. The majority of patients ( 90 percent) did not require an opioid medication refill. Among those with residual opioid mediations, most (59 percent) did not dispose of the remaining medication.

\section{DISCUSSION}

We demonstrate notable variations in practice among clinicians prescribing opioid medications for acute pain management in children and adolescents. We also demonstrate a high prevalence of 


\begin{tabular}{|c|c|c|c|}
\hline \multicolumn{4}{|c|}{ Table 1. Clinician survey questions and responses $(N=190)$} \\
\hline \multirow{2}{*}{ Questions } & \multicolumn{3}{|c|}{ Responses } \\
\hline & $\mathbf{N}$ respondents & $\mathbf{N}($ percent $)$ & Answer categories \\
\hline \multirow{4}{*}{$\begin{array}{l}\text { How do you assess acute pain in your } \\
\text { patients? Select all that apply. }\end{array}$} & \multirow{4}{*}{185} & $135(73)$ & $\begin{array}{l}\text { By patient self-report of presence or } \\
\text { absence of pain }\end{array}$ \\
\hline & & $140(76)$ & Using a numeric pain scale $(0-10)$ \\
\hline & & $86(46)$ & Using FACES pain scale \\
\hline & & $38(21)$ & Other \\
\hline \multirow{5}{*}{$\begin{array}{l}\text { What types of non-narcotic based acute pain } \\
\text { management do you provide? Select all that } \\
\text { apply. }\end{array}$} & \multirow{5}{*}{188} & $138(73)$ & Comforting/distraction measures \\
\hline & & $182(97)$ & $\begin{array}{l}\text { Non-narcotic pain medications (Tylenol, } \\
\text { NSAIDs) }\end{array}$ \\
\hline & & $26(14)$ & Antidepressants \\
\hline & & $53(28)$ & $\begin{array}{l}\text { Alternative/complementary treatments } \\
\text { (hypnosis, acupuncture, etc.) }\end{array}$ \\
\hline & & $21(11)$ & Other \\
\hline \multirow{2}{*}{$\begin{array}{l}\text { Do you use narcotic medications to manage } \\
\text { acute pain? }\end{array}$} & \multirow{2}{*}{185} & $118(64)$ & Yes \\
\hline & & $67(36)$ & No \\
\hline \multirow{12}{*}{$\begin{array}{l}\text { At what level of acute pain on a scale of } 0 \text { to } \\
10 \text { do you dose narcotic medications for pain? }\end{array}$} & \multirow{12}{*}{102} & $0(0)$ & 0 \\
\hline & & $0(0)$ & 1 \\
\hline & & $0(0)$ & 2 \\
\hline & & $0(0)$ & 3 \\
\hline & & $5(5)$ & 4 \\
\hline & & $12(12)$ & 5 \\
\hline & & $10(10)$ & 6 \\
\hline & & $24(24)$ & 7 \\
\hline & & $15(15)$ & 8 \\
\hline & & $3(3)$ & 9 \\
\hline & & $1(<1)$ & 10 \\
\hline & & $32(31)$ & $\begin{array}{l}\text { I don't use a scale. I dose on clinical } \\
\text { expertise and impression of patient pain }\end{array}$ \\
\hline \multirow{2}{*}{$\begin{array}{l}\text { Do you refill prescriptions for narcotics for } \\
\text { acute pain? }\end{array}$} & \multirow{2}{*}{103} & $26(25)$ & Yes \\
\hline & & $77(75)$ & No \\
\hline \multirow{3}{*}{$\begin{array}{l}\text { If you answered yes to refilling narcotic pre- } \\
\text { scriptions for acute pain, do you reassess pain } \\
\text { before refilling? }\end{array}$} & \multirow{3}{*}{26} & $21(81)$ & Yes, via patient report of pain \\
\hline & & $5(19)$ & Yes, via pain rating scale \\
\hline & & $0(0)$ & No \\
\hline
\end{tabular}


Table 1. Clinician survey questions and responses $(\mathrm{N}=190)$ (continued)

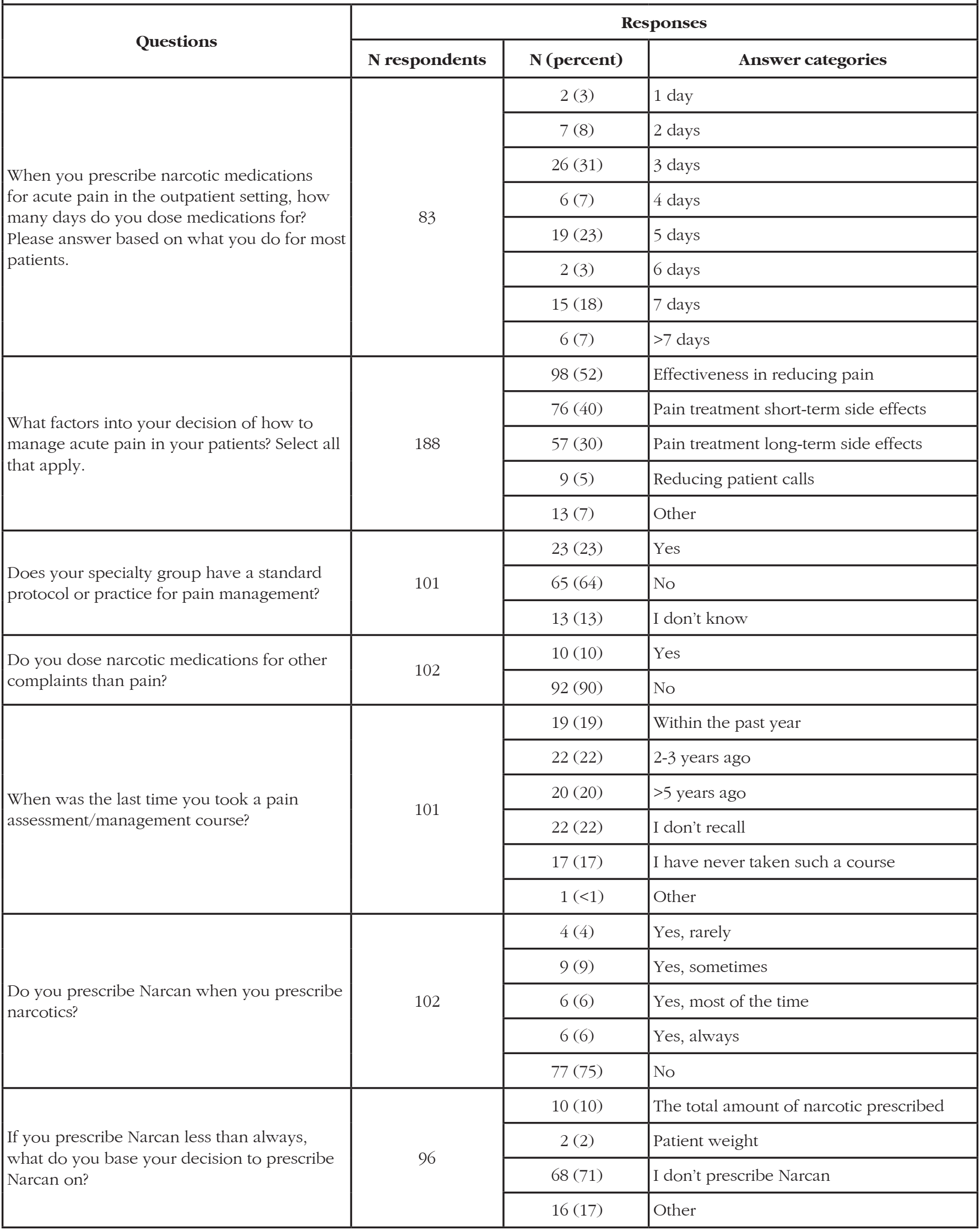




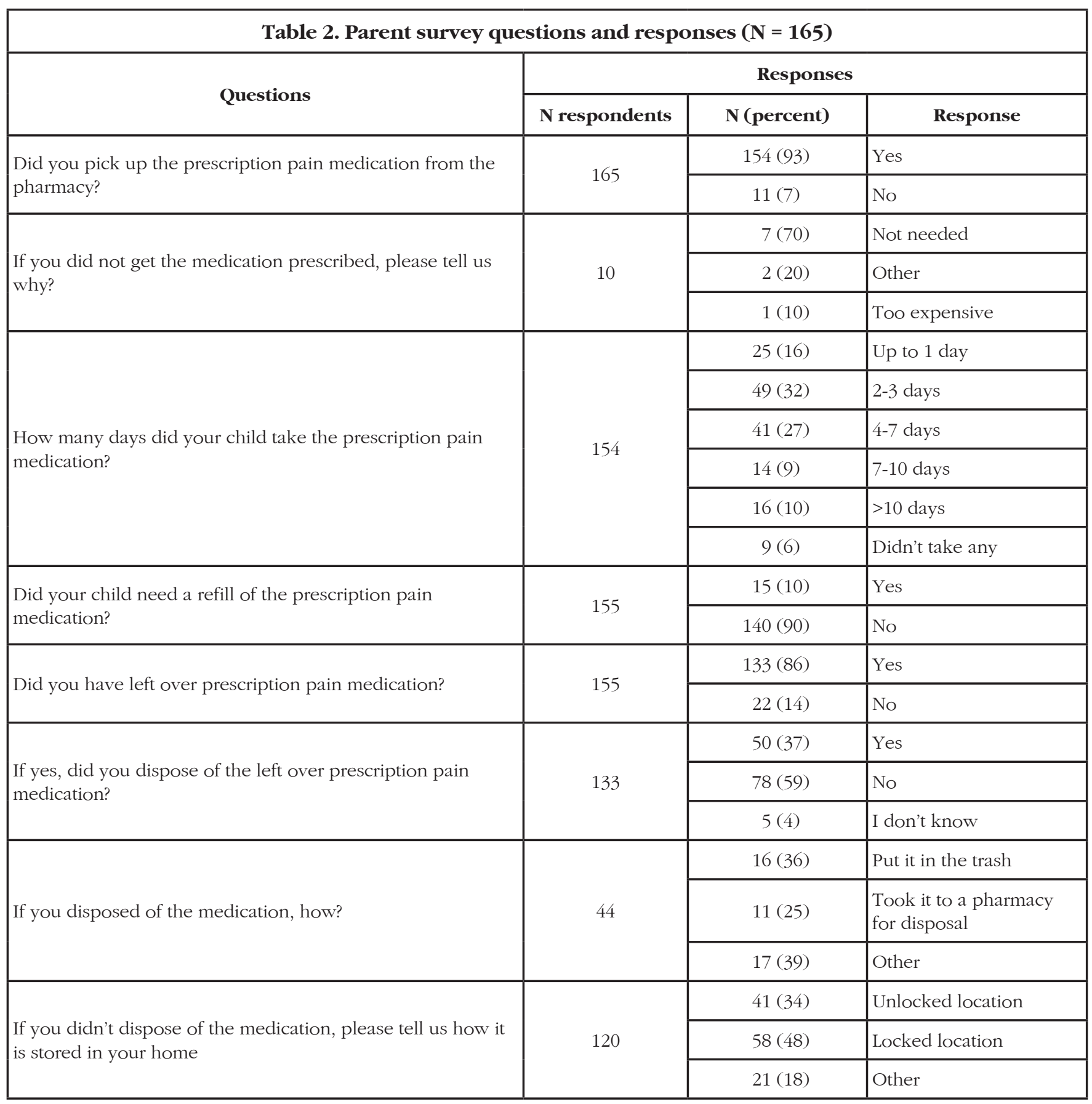

leftover medications (86 percent) among pediatric patients whose parents filled opioid prescriptions. These data demonstrate the need for standardized protocols to reduce patient risk associated with opioid medication exposure in the vulnerable pediatric population.

The Centers for Disease Control (CDC) published best practice recommendations on prescribing opioids for management of acute and chronic pain in adults. ${ }^{9}$ Similar recommendations do not currently exist in children, and there are sparse data available to inform future recommendations. While some admonish the application of adult-specific guidelines to children, ${ }^{10}$ the recommendation by the CDC that opioid prescriptions for acute pain management be limited to 3 days' duration is supported by our data demonstrating that almost half ( 48 percent) of surveyed pediatric patients prescribed opioids for acute pain management take it for 3 days at most. Nevertheless, we acknowledge that there are certain 
pain conditions in children (eg, spinal surgery in children $^{11}$ and sickle cell disease ${ }^{12}$ ) that may warrant more prolonged opioid prescriptions to manage pain.

Another recommendation by the CDC is that clinicians should continue opioid therapy only if there is "clinically meaningful" improvement in pain and function. Interestingly, in our clinician cohort, there were different clinical practices seen for initiation of as compared to continuation on opioid therapy. Most reported first using quantitative pain scales to assess pain and requiring a minimum pain score of 7 out of 10 or higher to initiate opioid medications. However, in regards to determining whether to continue on therapy, surveyed clinicians relied primarily on patient qualitative (whether still present or relieved) instead of quantitative (on the scale of 0 to 10) report of pain.

Almost one in three physician respondents to our survey reported relying on clinical expertise and impression to determine patient pain levels rather than quantitative pain scores. Quantification of pain is necessary to determine whether pain is worsening or whether a given therapy is providing effective relief from pain. Accordingly, quantitative pain rating scales (both self-report and proxy observer) have been developed for and validated in children across the cognitive and developmental age spectra ${ }^{13}$ and are commonly used in clinical settings. Some have argued that pain severity scales only capture a "very narrow aspect of a patient's pain experience" and that these scales should not be used to make opioid decisions. ${ }^{14}$ Others have stated that using pain scores alone to titrate dosage or dosing of opioid medications is "risky and inappropriate." 15 We submit that pain intensity scores provide valuable information regarding pain (particularly, improvement or worsening) and should not be discarded but rather supplemented with other clinically feasible measures of functional outcomes. One such measure, the Pain, Enjoyment of Life, and General Activity (PEG) scale, which assesses pain intensity and interference has been shown to provide a multidimensional measurement of pain that is clinically feasible, valid, responsive, and reliable in adult patient populations. ${ }^{16}$ An adaptation for pediatric populations might provide similar utility for comprehensive pain assessment that enables more effective pain management.

Other factors may also affect clinical decisions to dose opioids for pain relief. A minority ( 5 percent) of our clinician respondents also indicated patient satisfaction (inferred from "reducing patient calls") as a motivating factor to prescribe opioid medications. Similarly, in prior work, clinicians have reported prescribing opioids unnecessarily to improve patient satisfaction scores. ${ }^{17}$

The CDC recommends considering prescription of naloxone to mitigate the risk associated with opioid dosing. In our cohort, only 25 percent of clinicians reported prescribing naloxone with an opioid prescription. In contrast, acceptability by primary care providers for coprescription of naloxone with long-term opioid therapy for pain in adults has been demonstrated to be relatively high (79-83 percent). ${ }^{18,19}$ The overall low risk to benefit ratio of home prescription of naloxone must be considered in light of the opioid epidemic and prevention of morbidity. Nevertheless, guidelines regarding the dosing of naloxone in the pediatric setting have yet to be established and the majority of our clinicians did not report prescribing naloxone when prescribing opioids. An additional consideration for prescription of naloxone in children who receive opioids is that certain youth appear to be at greater risk for opioid-related adverse events. In particular, children with sleep-disordered breathing may have increased sensitivity to opioids leading to significant respiratory depression, anoxic brain injury, and even death. ${ }^{20}$ While some of this risk has been attributed to potential genetic factors, ${ }^{21,22}$ suggesting that genetic screening might help identify those at risk, the practical approach of generally reducing opioid dosing and using alternative medications to opioids has been shown to reduce the incidence of respiratory events requiring intervention. ${ }^{23}$

While our study did not address risk factors for opioid addiction, recognized risk factors do exist, including past alcohol, cocaine, and drug abuse, prior drinking under the influence (DUI) conviction, ${ }^{24}$ major depression, ${ }^{25}$ and psychotropic medication use. In addition, shortening of the time from first opioid use to opioid dependence in adulthood has been observed with presence of conduct disorder and child maltreatment (severe physical abuse) in childhood ${ }^{26}$ Identification of risk factors for opioid addition should be attempted prior to prescription of opioid medications, particularly in the setting of longterm prescriptions and at time of requested refill.

Another best practice commonly recommended for medications with adverse event profiles including opioids is that unused medication should be properly discarded. We demonstrate that 86 percent 
of families surveyed had leftover medication following opioid prescription for acute pain. This rate is similar to that reported in a prior report examining opioid therapy after elective pediatric procedures. ${ }^{27}$ The high prevalence of leftover opioid medication is particularly relevant given demonstrated ingestion of other persons' opioid medications by youth and adolescents in opioid misuse. ${ }^{28}$

Options available to families for discarding medications safely include drug disposal bins, pharmacy take-back, and mixing of medications with inactivating agents such as coffee grounds and charcoal. However, as noted in our parent survey, most families tend to keep their opioid medications rather than discard them, as has been reported among Medicare patients. ${ }^{29}$ Of those who kept the medication, most (48 percent) reported storing these unused medications in a locked location but many (34 percent) did not. A prior survey of adults who used a prescription opioid medication in the past year demonstrated suboptimal safe storage rate percentages that, depending on age of children in the home, ranged from 12 to 33 percent. ${ }^{30}$ Recent intervention work demonstrates that patient education efforts (particularly discussing risks of painkiller addiction) may reduce self-reported saving of pills. ${ }^{31}$ At the time of our parent survey, our institution did not routinely provide patient education regarding storage and/ or disposal of opioid medications; we are currently instituting a patient education campaign on these important topics as a result of this work.

Limitations of our work include the limited response rate of invited clinicians and parents, which may reduce generalizability of our findings. Nevertheless, responses are in line with what limited data are available in the literature and also agree with previously reported prescribing data from our institution. Specifically, at our institution, in the inpatient arena from 2015 to 2016, patients received opioids a median (interquartile range) of $1(1,2)$ days and a mean (SD) of 3 (10) days. ${ }^{32}$ In the ambulatory care setting, surgical specialties prescribed opioids an average of 7 (10) [mean (SD)] days while medical specialties prescribed opioids an average of 5 (6) days. ${ }^{33}$ Future research and data are needed to provide additional evidence for proposed practice recommendations.

In conclusion, our data demonstrate notable variability in clinician prescribing practices for acute pain management in children and overprescribing of opioids by clinicians. Our findings support the need to reduce extraneous and unnecessary exposure of a vulnerable population to opioid toxicities through quality improvement projects targeting clinician education and drug prescribing, development of standardized prescribing guidelines, and patient opioid utilization and disposal monitoring.

Jeannie S. Huang, MD, MPH, Professor, Department of Pediatrics, University of California San Diego, San Diego, California; Director, Clinical Medical Education, Rady Children's Hospital, San Diego, California.

Cynthia L. Kuelbs, MD, Professor, Department of Pediatrics, University of California San Diego, San Diego, California; Chief Medical Information Officer, Department of Biomedical Informatics, Rady Children's Hospital, San Diego, California.

\section{REFERENCES}

1. Johnston LD, O'Malley PM, Meich RA, et al.: Monitoring the Future National Survey Results on Drug Use, 1975-2015: Overview, Key Findings on Adolescent Drug Use. Ann Arbor, MI: University of Michigan, 2016.

2. Epstein-Ngo QM, Walton MA, Chermack ST, et al.: Eventlevel analysis of antecedents for youth violence: Comparison of dating violence with non-dating violence. Addict Behav. 2014; 39(1): 350-353.

3. Yazdy MM, Desai RJ, Brogly SB: Prescription opioids in pregnancy and birth outcomes: A review of the literature. J Pediatr Genet. 2015; 4(2): 56-70.

4. Sirnes E, Oltedal L, Bartsch H, et al.: Brain morphology in school-aged children with prenatal opioid exposure: A structural MRI study. Early Hum Dev. 2017; 106-107: 33-39.

5. Miech R, Johnston L, O'Malley PM, et al.: Prescription opioids in adolescence and future opioid misuse. Pediatrics. 2015; 136(5): e1169-e1177.

6. McCabe SE, West BT, Veliz P, et al.: Trends in medical and nonmedical use of prescription opioids among US adolescents: 1976-2015. Pediatrics. 2017; 139(4): e20162387.

7. Harbaugh CM, Lee JS, Hu HM, et al.: Persistent opioid use among pediatric patients after surgery. Pediatrics. 2018; 141(1): e20172439.

8. Naji L, Dennis BB, Bawor M, et al.: The association between age of onset of opioid use and comorbidity among opioid dependent patients receiving methadone maintenance therapy. Addict Sci Clin Pract. 2017; 12(1): 9.

9. Dowell D, Haegerich TM, Chou R: CDC Guideline for Prescribing Opioids for Chronic Pain-United States, 2016. MMWR Recomm Rep. 2016; 65(RR-1): 1-49.

10. Schechter NL, Walco GA: The potential impact on children of the CDC guideline for prescribing opioids for chronic pain: Above all, do no harm. JAMA Pediatr. 2016; 170(5): 425-426.

11. Wade Shrader M, Nabar SJ, Jones JS, et al.: Adjunctive pain control methods lower narcotic use and pain scores for patients with adolescent idiopathic scoliosis undergoing posterior spinal fusion. Spine Deformity. 2015; 3(1): 82-87. 
12. Okorji LM, Muntz DS, Liem RI: Opioid prescription practices at discharge and 30- day returns in children with sickle cell disease and pain. Pediatr Blood Cancer. 2017; 64(5).

13. Beltramini A, Milojevic K, Pateron D: Pain assessment in newborns, infants, and children. Pediatr Ann. 2017; 46(10): e387-e395.

14. Voepel-Lewis T, Malviya S, Tait AR: Inappropriate opioid dosing and prescribing for children: An Unintended consequence of the clinical pain score? JAMA Pediatr. 2017; 171(1): 5-6.

15. Voepel-Lewis T, Malviya S: Pain score guided morphine titration is risky and inappropriate. Paediatr Anaesth. 2014; 24(4): 454-456.

16. Krebs EE, Lorenz KA, Bair MJ, et al.: Development and initial validation of the PEG, a three-item scale assessing pain intensity and interference. J Gen Intern Med. 2009; 24(6): 733-738.

17. Zgierska A, Rabago D, Miller MM: Impact of patient satisfaction ratings on physicians and clinical care. Patient Prefer Adherence. 2014; 8: 437-446.

18. Behar E, Rowe C, Santos GM, et al.: Acceptability of naloxone co-prescription among primary care providers treating patients on long-term opioid therapy for pain. J Gen Intern Med. 2017; 32(3): 291-295.

19. Behar E, Rowe C, Santos GM, et al.: Academic detailing pilot for naloxone prescribing among primary care providers in San Francisco. Family Med. 2017; 49(2): 122-126.

20. Kelly LE, Rieder M, van den Anker J, et al.: More codeine fatalities after tonsillectomy in North American children. Pediatrics. 2012; 129(5): e1343-e1347.

21. Voronov P, Przybylo HJ, Jagannathan N: Apnea in a child after oral codeine: A genetic variant-An ultra-rapid metabolizer. Paediat Anaesth. 2007; 17(7): 684-687.

22. Voelker R: Children's deaths linked with postsurgical codeine. JAMA. 2012; 308(10): 963.

23. Raghavendran S, Bagry H, Detheux G, et al.: An anesthetic management protocol to decrease respiratory complications after adenotonsillectomy in children with severe sleep apnea. Anesth Analg. 2010; 110(4): 1093-1101.

24. Ives TJ, Chelminski PR, Hammett-Stabler CA, et al.: Predictors of opioid misuse in patients with chronic pain: A prospective cohort study. BMC Health Serv Res. 2006; 6: 46.

25. Boscarino JA, Rukstalis M, Hoffman SN, et al.: Risk factors for drug dependence among out-patients on opioid therapy in a large US health-care system. Addiction. 2010; 105(10): 1776-1782.

26. Sartor CE, Kranzler HR, Gelernter J: Rate of progression from first use to dependence on cocaine or opioids: A cross-substance examination of associated demographic, psychiatric, and childhood risk factors. Addict Behav. 2014; 39(2): 473-479.

27. Voepel-Lewis T, Wagner D, Tait AR: Leftover prescription opioids after minor procedures: An unwitting source for accidental overdose in children. JAMA Pediat. 2015; 169(5): 497-498.

28. Substance Abuse and Mental Health Services Administration: Results from the 2013 National Survey on Drug Use and Health: Summary of National Findings. Rockville, MD: Substance Abuse and Mental Health Services Administration, 2014.

29. Maeng DD, Snyder RC, Medico CJ, et al.: Unused medications and disposal patterns at home: Findings from a Medicare patient survey and claims data. J Am Pharm Assoc. 2016; 56(1): 41-46.

30. McDonald EM, Kennedy-Hendricks A, McGinty EE, et al.: Safe storage of opioid pain relievers among adults living in households with children. Pediatrics. 2017; 139(3).

31. Hero JO, McMurtry C, Benson J, et al.: Discussing opioid risks with patients to reduce misuse and abuse: Evidence from 2 surveys. Ann Family Med. 2016; 14(6): 575-577.

32. Bagot K, Billman G, Chiang G, et al.: An EPIC opportunity to improve opioid prescription practices for children. Paper presented at: Interprofessional Innovations 2017, San Diego, CA.

33. Huang JS, Kuelbs C: Pediatric opioid prescribing practices at a large metropolitan children's hospital. Paper presented at: Pediatric Academic Societies 2017, San Francisco, CA. 